This a rough draft not to be quoted or circulated. Comments would be appreciated. -Gabriel Segal

\title{
Poverty of Stimulus Arguments Concerning Language and Folk Psychology
}

\section{$\underline{0 . \text { Introduction }}$}

This paper is principally devoted to comparing and contrasting poverty of stimulus arguments for innate cognitive apparatus in relation to language and in relation to folk psychology. These days one is no longer allowed to use the term 'innate' without saying what one means by it. So I will begin by saying what I mean by 'innate'. Sections 2 and 3 will discuss language and theory of mind, respectively. Along the way, I will also briefly discuss other arguments for innate cognitive apparatus in these areas.

\section{What is it for a cognitive capacity to be innate?}

When Noam Chomsky argues for innateness of linguistic knowledge, one can discern two aspects of the notion he is working with. The actual arguments are aimed at a negative conclusion about how linguistic knowledge is acquired; they are arguments about how it is not acquired. Let us consider a typical case (from Chomsky 1986):

(1) a. John ate an apple

b. John ate

c. John is too clever to catch Bill

d. John is too clever to catch

(1b) is typically understood to mean: 'John ate something or other edible'. Thus looking at (1a) and (b) one might conclude that if a verb that has both transitive and intransitive forms appears without a syntactic object, then interpret it as having an implicit arbitrary object of appropriate category. But if you apply that rule to (1d) you get the conclusion that it means that John is too clever to catch something or 
other catchable. And of course it doesn't mean that. It means that John is too clever for one to catch him.

Why do we follow rules that mandate that particular interpretation of that sentence? How come typical English speakers interpret (1d) in the way we do rather than some other way - for example, on the model of (1b)? Do we learn the rules by some standard sort of inference from data, some sort of analogical reasoning, induction, abduction, statistical inference or deduction? Chomsky says: 'no'. Any standard form of reasoning would likely find a rule that applies to simpler sentences, like (1a) and (b), then apply it to the more complex cases. But that is not what we do. Further, we were not taught how to interpret sentences like (1d). And, although Chomsky doesn't bother to point this out, we obviously do not simply perceive the rule. So we did not learn the rule in any normal sense of learning. Or, to put it more generally and more helpfully, (following Richard Samuels (2002)): knowledge of the rule is psychologically primitive, i.e. it is not acquired by any psychological process, by any process of perception or inference.

But Chomsky sometimes adds a positive gloss, a hint about how we do acquire knowledge: it is 'biologically determined', 'genetically determined', 'part of our biological endowment' 'on a par with elements of our common nature that cause us to grow arms and legs rather than wings' (Chomsky 4).

But what do those locutions mean? The growth of arms and legs is of course not literally genetically determined, in the sense that having a certain genetic structure suffices, all by itself, for growth of the limbs. Obviously, the environment has to be amenable as well. One way of tidying up the positive notion of innateness would be just to stop with something like: growth in the manner of limbs and organs. Samuels expresses it in the terms: 'acquired in some normal way'. So, if our knowledge of linguistic rules was acquired as a result of, say, brain-programming by alien scientists, then the acquisition of rules would not be via a psychological process. But it would not count as innate, since it would not be the result of biologically normal growth.

Samuels's account seems good to me. But I think that if one's concern is debates about acquisition in cognitive psychology, then it is not worth spending too much energy trying to articulate a proper account of the positive aspect of innateness.

The work is done by the negative clause: 'not acquired by a psychological process'. Thus empiricists propose that their chosen explanandum is acquired by: induction, abduction, deduction, perception, statistical inference, conditioning etc... Nativists, say: no, it was acquired some other way. It has to be considered primitive from the psychological point of view - something that we assume that the learner has before the process of learning begins. If alien scientists programmed us with UG when we were foetuses, then I take it that nativists come out as more nearly right than empiricists.

So Samuel's account seems good, especially if we don't worry too much about the second clause. But there is another notion of innateness that also seems healthy and that could be seen to capture the ideas roguhoy expressed by 'genetically determined' and the like: it is a version of canalization (Waddington 1940). A trait is canalized to the extent that its development is causally insensitive to environmental and genetic variation. So, for example, the development of fingers is highly canalized, since it is relatively insensitive to the relevant influences. Development of skin colour is less so, since it is causally affected 
by exposure to sunlight. We can think of a trait as innate to the extent that it is developmentally canalized.

Fiona Cowie has offered two objections to this construction of the notion of cognitive innateness.

The first objection is that the debate between nativists and empiricists is not happily seen as one about the extent to which cognitive development is canalized. The reason for this is that the extent to which development is canalized depends on which range of environments one considers (Cowie 1999 45-6). So, for example, if you are considering all environments capable of sustaining human life, then development of normal facial features looks highly canalised, since these features develop in a very wide range of hominid environments. But if you are an obstetrician interested in the healthy development of normal babies, then you might focus on a range of in utero environments many of which interfere with normal facial development. Development of a normal face will then appear not to be highly canalized. So empiricists and nativists seem merely do be diverging over the choice of which environments to consider. Empiricists focus on a narrow range of environments in which environmental factors tend to affect development, while nativists consider a wide range of environments most of which do not contain influential factors.

But Cowie's concern is unfounded. Different parties may disagree on the causal role of environmental factors that are present within a given range of environments. And that is indeed the nature of the disagreement between nativists and empiricists. They all consider mundane, ordinary environments, and they disagree over the nature of the developmental process that unfolds within them. Empiricists say development is causally sensitive to environmental factors, nativists say it isn't. Consider, for example, the issue over the rules for interpreting (1d). An empiricist would say that these are acquired by some learning process, say by induction from other examples. A learning process, such as induction, is precisely one that is causally sensitive to environmental specifics. Nativists say: no, the acquisition process is insensitive to environmental factors. Knowledge of the rule grows in the head, it doesn't get put there by a process that examines the environment and responds to it.

Cowie's second objection is that poverty of stimulus arguments are not arguments to the effect that the acquisition process is highly canalized. But actually they can easily be understood in that light. The relevant range of environments for the debate are those where some linguistic stimuli are present and different stimuli are present in different environments. If the developmental process results in acquisition of the relevant feature even in environments where stimulus is poor, then we can infer that the developmental process is not causally sensitive to the environment. The process doesn't require anything much from the environment: just decent physical and psychological living conditions and some linguistic stimulation. That is to say, the relevant pair of options to consider are: developmental processes that respond differentially to differences in experience and those that don't. The former require rich stimuli, the latter do not.

In support of her argument, Cowie (46) cites Plato's slave boy who comes up with a relatively sophisticated belief about geometry on the basis of just a little questioning from Socrates. In this case, the stimulus is impoverished, but the outcome is not highly canalized, since it will only occur in a narrow range of environments. But there are two cognitive properties to consider, not one. One is the specific 
geometric belief elicited from the slave boy. Its development, as Cowie says, is not highly canalised. But nor should it be considered innate. (Note also that it is acquired by a psychological process of inference, hence that it is not psychologically primitive). The second is Universal Geometry, the geometrical equivalent of Universal Grammar, (roughly speaking) whatever general knowledge of figures enabled the slave boy to deduce the geometric facts under the influence of suitable questioning. Universal Geometry is innate and its development is highly canalized.

So canalization does look like a good interpretation of nativists' informal talk of 'genetic determination' and the like.

How, then, do canalization and primitiveness relate? I think it is reasonable to hypothesise that the amongst cognitive properties development of all and only the primitives ones will be highly canalized. After all, psychological non-primitives are all acquired by psychological processes: perception, induction, abduction and the rest. And, as I said above, these processes do not canalise their outcomes: the whole point of these processes is that they are designed to be causally sensitive to environmental contingencies. It is less clear that cognitive primitives will all be canalized. There is no obvious reason to suppose that development of a primitive trait couldn't be significantly influenced by environmental factors. However I am not aware of any examples of this.

So, in sum: the notions of innateness appropriate for cognitive psychology are 1. Samuel's primitiveness and 2. Waddington's canalization. And it is reasonable to hypothesise that these are largely co-extensive.

I move on now to look at poverty of stimulus arguments, beginning with their application to knowledge of language.

2. What poverty of stimulus?

Poverty of stimulus arguments have held a central place in linguistics and cognitive psychology for the last thirty years or so. But they are surrounded by controversy. There is controversy about how they should be formulated and there is controversy about how powerful they are when deployed in relation to this or that or any item of knowledge.

I will begin by stating the basic form of a poverty of stimulus argument in as simple and general a way as possible along with a more specific version that applies to linguistic knowledge in particular.

POSBASIC

For a given psychological property, $\mathrm{P}$ :

a. Some significant group of cognizers have $\mathrm{P}$

b. Cognizers acquire P either via general-purpose learning mechanisms or with the aid of an innate 
domain-specific cognitive endowment.

c. Stimuli are too poor for the cognizers to acquire $\mathrm{P}$ by general-purpose learning methods.

so

d. Cognizers do not acquire $\mathrm{P}$ by general-purpose learning methods

so

e. Cognizers acquire $\mathrm{P}$ with the aid of a domain-specific cognitive endowment.

And for the specific case of linguistics we have:

POSLING

For a given item of syntactic knowledge, K:

a. Some significant group of children unerringly acquire $\mathrm{K}$

b. Children acquire K either via general-purpose learning mechanisms or with the aid of innate domainspecific knowledge.

c. Children's stimuli are too poor to provide them with data from which they could learn $\mathrm{K}$ by generalpurpose learning methods.

so

d. The children do not learn $\mathrm{K}$ by general-purpose learning methods

so

e. The children acquire $\mathrm{K}$ with the aid of innate domain-specific knowledge

A few comments are in order. 'Knowledge' is used in the standard cognitive psychological sense to mean, roughly, representational content. There is no commitment to the view that knowledge must be explicitly represented. 'Syntactic knowledge' is used in a broad sense in which Chomsky sometimes uses it, to apply to syntactic and, possibly, what some would call 'semantic' knowledge. 'General-purpose learning methods' (also called 'empiricist' (Laurence and Margolis 2001) or 'data-driven learning'(Pullum and 
Scholz 2002) covers the usual empiricist kit bag of induction, abduction and so on.

Premise b. of POS ${ }^{L I N G}$ assumes that the innate endowment relevant to language acquisition includes some syntactic knowledge. Thus it is assumed that special-purpose learning may just combine some general-purpose method with some primitive syntactic knowledge. The argument would thus be rendered unsound by the existence of special-purpose learning mechanisms that do not deploy innate syntactic knowledge, such as learning by a PDP system with innate biases that do not realise syntactic knowledge.

Let us look at a relatively simple example, so-called 'Binding Principle (A)'.

Please consider (1):

(1) Ann wanted Mary to shake hands with herself

Speakers typically take this to mean that Ann wanted Mary to shake hands with Mary. They do not take it to mean that Ann wanted Mary to shake hands with Ann, although the latter interpretation would usually be more plausible on pragmatic grounds. A plausible explanation of the rules determining these constraints on interpretation goes like as follows. The underlying syntactic form of (1) is (2):

(2) $\mathrm{Ann}_{\mathrm{j}}$ wanted Mary $\mathrm{k}$ to shake hands with herself $\mathrm{k}_{\mathrm{k}}$

The subscripts are real but hidden elements of the sentence. Co-indexed expressions stand in a syntactic relation called 'binding': the pronoun 'herself' is bound by the name 'Mary'. And they determine referential possibilities for the pronoun: co-indexed expressions must co-refer. The reason that 'Ann' and 'herself' cannot be co-indexed is that there is too much linguistic material between them. The notion of 'too much linguistic material' can be made precise, and is relatively technical and complicated.

Binding Principle (A) informally specifies this rule governing the binding of reflexives:

(A) Reflexives must be locally bound.

The explanation of why adults interpret sentences like (1) the way they do is that they tacitly know Binding Principle (A). And children are like adults in respect of how they interpret sentences like (A). They never make mistakes about the interpretation of reflexives in such contexts. So it appears that they unerringly acquire Binding Principle (A).

There are examples of more complex and sophisticated syntactic rules that children appear to be following, such as "negative polarity items are licensed in downward-entailing environments". But there is no need for me to articulate any here. (See e.g. Pietroski and Crain (2002) for examples). And the more complicated and sophisticated $\mathrm{k}$ is, the more compelling POS ${ }^{\mathrm{LING}}$ gets. 
Let us now return to POSLING and the crucial premise c: "Children's stimuli are too poor to provide them with data from which from which they could learn $\mathrm{K}$ by general-purpose learning methods". Premise $\mathrm{c}$ is supported by the following three considerations.

The first supporting consideration just brings out the point of the inclusion of the term "unerringly" in premise a. One would expect a general-purpose learning mechanism to err as it acquired an item of knowledge such as Binding Principle (A). That is to say if children were learning binding principle say by induction or abduction we would expect a reasonable percentage of them to get it wrong before getting it right. But that is not what we find.

The second supporting consideration is that we can easily describe alternative sets of grammatical rules that are consistent with the linguistic data available to children and that are a priori as just as plausible as the actual rules of Universal Grammar, but that children never come up with. For example, we can describe a language in which all pronouns have their reference pragmatically determined, and that has a loose, ceteris paribus conventional implicature to the effect that reflexives should be used to co-refer with nearby subjects.

The third supporting consideration is that we can use linguists as a crude measure of the difficulty of learning syntactic rules using general-purpose methods.

Linguists have access to lots of secondary linguistic data that assists them with their inquiries: data from different languages, data concerning childrens' patterns of acquisition, data from neural deficits and so on. And linguists work in groups. They can read each others' books and articles. And yet each child, working alone, tacitly knows far more about the rules of Universal Grammar than does any linguist.

A possible empiricist counter to this last point would be that although linguists deploy general-purpose learning mechanisms, they do not deploy the same ones that children do. For example, an empiricist might claim that language is acquired by a parallel distributed processing network that in effect does sophisticated and subtle statistical analysis. Since linguists are not using the same method, their relative ignorance is irrelevant. On the other hand some relatively empiricist psychologists argue that children's acquisition mechanisms use just the same sorts of statistical analysis as scientists' (See Shultz and Gopnik 2004). This counter would obviously not be open to them.

There is a further point to be made in favour of POS ${ }^{L I N G}$. The conclusion of the argument is corroborated by the empirical research that nativist linguists have carried out. There are detailed proposals about some of the properties of Universal Grammar that address a wide array of data with precision and in detail, including data concerning patterns of acquisition linguistic universals and deficit data. A cogent empiricist counter would require a similarly detailed and well-supported theory. We are still waiting

I conclude that POSLING is in very good shape. 


\subsection{The Evidence for Linguistic Nativism Stands Up}

There are of course many arguments for nativism in linguistics other than poverty of stimulus. I shall mention just two, which I take to be the most compelling.

The first is the argument from Creoles and sign languages. Creoles and certain sign languages developed by individuals or groups have been found to conform to the same grammatical principles as other natural languages (ref Pinker).

Cowie floats two possible empiricist accounts of these data (although she places little confidence in them). The first suggestion is that people develop languages that way because it facilitates communication. There are at least two problems with that proposal. First, the idea that rules of natural language are particularly well suited for facilitating communication is really very implausible. One of the striking points about universal grammar is precisely that it seems arbitrary from the point of view of communicative efficiency. But even if it is true that grammars conforming to UG are very efficient communication systems, they are surely not the only ones. Thus languages that do not conform to UG such as languages based on formal logic - can be just as good. So even given the idea that people home in on efficient systems when they develop natural languages, one still needs to explain why they home on those conforming to UG rather than alternatives.

The second problem is deeper. The question is: what cognitive resources are required to get from the available input plus a desire to communicate efficiently to a language designed according to UG? Even if human languages are maximally efficient for communication, that does not begin to explain why we invent them. What kind of general-purpose language designer would come up with the weird and wonderful rules of Universal Grammar? Empiricists have a hard enough time explaining how a generalpurpose learning mechanism could come up with UG, even given as input a language conforming to UG. The task of coming up with one without such input is monumental.

Cowie's second response is: people 'just aren't 100\% accurate when they learn languages... sometimes they add structure'. But that suggestion does not even address the issue. The question is: why do people add that particular structure, when there are infinitely many other ones that they might equally have added. If it was just a question of inaccuracy, you'd predict that different new languages would have different structures from one another and from old ones.

The second most compelling area of evidence comes from dissociations. In particular, there are cases of subjects whose only cognitive impairment is linguistic. Acquired aphasias are the best cases. Here, a subject can have very severe - sometimes total - lack of syntactic abilities, yet be normal in other cognitive domains (for example the subject SA, studied by Rosemary Varley and her collaborators; see e.g. Varley 1998). Cowie dismisses these cases as irrelevant, in that they do not tell us how the aphasic subjects acquired their languages before they lost them. But they are directly relevant, since we can ask: why can't these subjects relearn language? They can learn other things. If there are such things as generalpurpose learning systems, then these subjects have them. But they cannot learn language. Thus, whatever 
it is that enables children to learn language is domain-specific.

There are also cases of developmental dissociations, in particular so-called 'Specific Language Impairment'. Cowie doubts the relevance of SLI because it is a heterogeneous and controversial category. That it is. Nevertheless there is a particular sub-group of individuals with SLI who have normal (or superior) cognitive profiles but severe specific syntactic impairments, so-called "G-SLI". The number of these subjects is estimated to be 3-6 per 1000. (van der Lely and Stollwerck. See also van der Lely, et al. (2004) and van der Lely et. al., (1998) for compelling evidence that G-SLI is a language-specific impairment).

\section{$\underline{\text { 3. Folk Psychology }}$}

By 'folk psychology' ('FP') I mean knowledge of the body of principles by which normal human beings understand humans and other minded systems; principles for explaining how psychological states (desires, beliefs, emotions etc.) are acquired, and how psychological states interact to explain actions. The idea that folk psychology is a good candidate for poverty of stimulus arguments is mentioned reasonably often. But the argument is rarely formulated and has never been spelled out in any detail (as far as I know).

What are the prospects of a compelling argument? As soon as the question is posed, an important difference between language and FP becomes salient. In the case of language, it is reasonably clear what it is that nativists claim to be innate: viz. at least, knowledge of syntactic principles (concerning tree structures and relations defined over them) rich enough to include or yield as consequences rules like Binding Principle (A) and many others that can be found in linguistics journals. There is nothing analogous for the case of FP. There is no paradigm candidate for the purported innate endowment.

So let me first address that difficulty by laying out a spectrum of possible views. I take it that all parties can agree that mature FP consists in (roughly speaking) possession of a system of concepts or representations such as belief, desire, fear, hope etc. and the capacity to deploy these concepts in explanations and predictions of action. But there are a variety of different positions one might reasonably take with respect to the question of which aspects of FP are innate, which I have appropriately labelled in terms of Indian dishes. The strength of the dish corresponds to the strength of the position.

(1) Nativism Tindaloo Balti : a sophisticated folk theory plus dedicated performance systems.

This was the position briefly defended in Segal (1995) and roughly corresponds to Chomsky's position in relation to language.

(2) Nativism Tindaloo: one or more sophisticated classical computers, with explicit representations of FP concepts. 
This position is just as strong as Nativism Vindaloo Balti, but is prepared in a different way. It is defended by Leslie (1994) Fodor (1987) and Baron-Cohen (1994).

(3) Nativism Vindaloo: at least some of the core concepts of FP along with dedicated performance systems.

This view is consistent with that of Nicholls and Stich (2003).

(4) Nativism Korma: recognizably FP precursor concepts along with general-purpose learning mechanisms.

The idea would be that there is some domain-specific conceptual FP endowment, but that the developmental process after birth involves general-purpose learning mechanisms, such as abduction. Wellman (ref)? Perner (ref)?

(5) Empiricism Thali: various relatively low-level mind-reading systems are innate, such as: emotion detectors, intentionality detectors, eye-direction detectors, action-parsers and, imitation (co-ordinating infants' own emotions and kinaesthetic sensations with expressions and gestures of others) along with general-purpose learning mechanisms.

What are the prospects for running a compelling poverty of stimulus argument in favour of one of the strong nativist positions gestured at above (let's call it a 'POS ${ }^{\mathrm{FP}}$ ')? The basic thought behind a POSFP is simply that FP concepts or representations are far too sophisticated to be acquired by general-purpose learning mechanisms, given the available stimuli. FP concepts are concepts of states with representational content and causal powers such that the latter can be predicted from the former. A general-purpose learning mechanism could never come up with concepts/representations like that on the basis of exposure to moving bodies - even with the help provided by the apparatus of Empiricism Thali. That's the basic nativist thought. And the basic empiricist counter is: 'oh yes it could'.

There is a problem with constructing a compelling POSFP: almost none of the supporting considerations that apply in the case of POSLING apply here.

The first supporting point in the case of language was that if general-purpose learning mechanisms acquired knowledge of syntactic rules, they would do so erringly. In the case of language, we have evidence that many rules, such as Binding Principle (A), are acquired unerringly. There appears to be no analogue in the case of FP. There is no known range of specific FP judgements that kids get right and right first time that would lead us to posit that some specific pieces of FP knowledge had to be there from the start.

The second supporting point was that is easy to envisage alternative sets of syntactic rules that are consistent with the primary linguistic data, so we can pose the question of why children never hit on those 
but always hit on UG. There is no analogous point to be made in relation to FP. Children could become associationists but that's about the only known alternative. It is a striking and often-noted fact that theory theorists have failed to specify in any great detail what the contents of children's and adults' tacit theory of mind are.

The third point of support was that we can use linguists as a crude measure of the difficulty of acquiring syntactic knowledge given the available stimulus. Here there is a rough analogue in the case of FP, but only if it is agreed that FP is a theory. So the point has some polemical force against for example Gopnik, who does hold such a view. According to Gopnik (e.g. Gopnik and Wellman 1992, Schultz and Gopnik 2004) the theory is simple enough for three-year-olds to be able infer it from the stimuli available to them. But if that's the case then one would have thought that highly intelligent and knowledgeable psychologists and philosophers who have access not only to the data available to the children, but to the children themselves, ought to be able to figure out what the theory is. But we haven't.

The fourth point of support was that the conclusion of POSLING is corroborated by empirical research in linguistics. There is no serious corroborative research in the case of FP. That is to say, there is no proposal about the nature of Universal Folk Psychology that addresses a wide array of data in detail and with precision.

It thus appears that while the crude thought that would motivate a POSFP seems compelling to many, it could be difficult to come up with an argument that carries polemical force.

There is, however, at least one feature of FP that bolsters the idea of a good POSFP. There is good evidence from anthropology and developmental psychology that FP is profoundly dualist. FP portrays minds as immaterial entities, entities not subject to physical laws. From anthropology we learn that that among the folk (those not trained in philosophy or science) belief in spirits that can be disembodied is universal (Boyer (2003)). And there is evidence from developmental psychology that children are born dualists: they do not expect people to be subject to physical laws (Bloom (2004), Kuhlmeier et. al. (2004) ).It seems to me that no general-purpose learning mechanism would come up with such a bizarre account of the explanation of behaviour: that the mechanism that underlies human behaviour, the locus of thoughts and feelings, is an immaterial soul. We would not have arrived at that belief were we not born with it.

I am tentatively inclined to suggest that the idea that we are born dualists is also supported by the stubbornness of dualism. Philosophers and others whose official academic view is one or other form of physicalism, remain the in the grip of folk dualism. That is to say, we come to the explicit belief that in fact mental properties are properties of material objects, such as brains. And yet we continue in our lay thinking to portray minds as immaterial. We can't shake off the idea that really we are ghostly inhabitants of our bodies who could exist perfectly well in their absence. It may be that the reason we cannot shake of our dualistic way of thinking is because it is innate and modular.

Whatever the prospects for POSFP, there is a considerable difficulty for the more empiricist positions that 
I gestured at above: autism. High-functioning subjects with autism or Asperger's Syndrome can be very good at learning anything other than FP. Their general-purpose learning mechanisms are intact. And yet they have tremendous difficulties learning bits of FP and they never get as good it as a normal ten-yearold. There are autistic subjects who do well on false belief tasks, and who have a decent grasp of basic belief-desire psychology. But even they show significant impairments in psychological understanding. It is not just that they remain impaired in respect of perceptual aspects of mind-reading, such as eyereading. They remain impaired in respect of very cognitive aspects of mind-reading. For example, BaronCohen et. al. (1999) studied three very high-functioning subjects with Asperger's Syndrome and all three of them state that they really have very little idea how even to begin to understand people. They find social situations largely unfathomable, and often lack understanding of how their behaviour will be perceived by others. Such understanding of social matters as they do have appears to have been learnt largely piecemeal, often simply by their having been told. What appears to be lacking is knowledge of a theoretical and abstract sort, knowledge that a general-purpose learning system ought to be able to acquire by diligent study of human behaviour.

The proponent of Empiricism Thali would either have to endeavour to explain autism in terms of a deficit in one or more of the Thali dished or appeal to attempts to explain autism in terms of a non-FP deficit, such as weak central coherence or executive function disorder. But neither of these lines is promising. I begin with the former.

The problem with the first option is that it looks incapable of accounting for the persistent nature of cognitive mind-reading anomalies in autism. If all that an autistic child were missing were low-level mindreading systems then we should predict that they would be delayed in acquisition of FP, but would end up with normal cognitive FP abilities. FP is abstract and theoretical in nature. The information that normal individuals acquire from low-level components of the mind-reading system should not be required for learning FP. If FP were normally acquired by general-purpose methods, then high-functioning autistic individuals ought to be able to get round their lack of input from low-level mind-reading apparatus and catch up. But they do not.

Let us consider some data from Happe (1994). Here is a story in which someone utters a falsehood. You have to figure out why.

Katie and Emma are playing in the house. Emma picks up a banana from the fruit bowl and holds it up to her ear. She says to Katie: 'Look, this banana is a telephone'.

Why does Emma say this?

Happe presented this and similar tasks autistic individuals who passed $1^{\text {st }}$ and $2^{\text {nd }}$ order mind-reading tests. These subjects were in the normal IQ range and all but one were over 16 . They did not do too badly on the tests, failing on average about one out of eight. But they did do significantly worse than normal adults. Now consider an incorrect reply to the question about Emma that was offered by one autistic subject: "She wanted to fool her." 
To explain Emma's saying that the banana is a telephone, one has to have some sort of concept of playing. Even if one sees no point in playing and never oneself indulges in play, it should not be very hard to learn the word "playing" denotes an activity that often involves sayings that are not true. Nor should it be particularly hard to remember this and be inclined to explain Emma's utterance by saying: "she is playing" or "she is pretending" or something similar. It should be possible to acquire such a concept just by observing people: watching them, listening to them. One does not need dedicated mindreading apparatus, such as eye-direction or affect detectors for that. To fail to come up with the right explanation of Emma's utterance is to fail a rather simple cognitive task.

The explanation offered by the autistic subject tells the same story. It is obviously very unlikely that Emma would try to fool Katie into thinking that the banana is a telephone. This is because a banana looks nothing like a telephone and so it is very unlikely that Emma would succeed in fooling Katie. It is very likely that Emma herself would know this. Ceteris paribus, people don't attempt actions that they think are going to fail. So it is very unlikely that Emma would try to fool Katie in this manner. Again the conceptual repertoire required to work this out is the sort that could be acquired by a student of human behaviour. No special information of the sort provided by low-level mind-reading systems should be required.

I cannot at this point offer a knockdown argument for the conclusion that a Thali deficit could not account for autism. For one thing we do not yet have much data concerning which low-level systems are impaired in autistic subjects. But prospects for a Thali account of autism look bleak.

I move on now to consider the prospects for a weak central coherence (Frith and Happe 1994, roughly a 'can't-see-the-wood-for-the-trees' problem) or executive function (Russell ed. ref, an inability to suppress a prepotent response) account of autism.

\section{$\underline{3.1 \text { Executive Function and Central Coherence }}$}

Nativists have made a number of objections to weak central coherence and executive function accounts. (See Baron-Cohen and Swettenham (1997) for a fairly thorough overview). In my view, at least three are cogent.

The first is that some very high-functioning individuals with Asperger's Syndrome show superior abilities in respect of Tower of Hanoi (one of the chief tests of executive function and central coherence) while failing a mindreading task that involves inferring a subject's mental state from their eyes (Baron-Cohen et. al. 1999). Only three individuals have been tested and shown to exhibit this profile. But they do appear to be clear cases of strong executive function and central coherence combined with weak mindreading.

The second is that autism involves deficits in rather basic mindreading tasks that appear not to tap executive function and central coherence, such as eye-reading and social-referencing.

The third requires some discussion. Leslie and Thaiss (1992) tested autistic subjects on the following out- 
of-date photograph task. Subjects are familiarised with a camera and its functions. They then witness a photograph being taken of a toy animal on a chair. While the photo is developing, the subject sees the animal being moved to a bed. The subjects are then asked what the photograph will show. Autistic subjects did well on this task (better even than normal subjects) correctly answering that the photo would show the animal on the chair. This task appears to require the same resources of executive function and central coherence as a standard Sally-Anne task.

However, Jim Russell (Russell ed. 294) has argued that the photograph task in fact places lighter demands on executive function than does the Sally-Anne task:

when the test question [in the photo task] is asked the subject is faced with two representations: a threedimensional representation (e.g. of a girl in a yellow dress - a doll) and a two-dimensional representation (e.g. a picture of a doll in a red dress). Is a three-dimensional representation more cognitively salient than a two-dimensional one? Possibly. But is the difference in salience as great as that between my true belief and another's false belief...?

Russell et. al. (1999) then developed a variant of the photo task designed to bring the level of executive difficulty into line with Sally-Anne. In their scenario, they use a coloured backdrop against which objects can be photographed. Subjects are familiarised with the camera, and witness a photo being taken of an object in front of the screen. They get to see the developed picture. In the task, a photograph is taken of the blank screen with no object in front of it. While the picture is developing, a Barbie doll is placed in front of the screen. Children are then asked what the photo will show. Autistic subjects did not cope well with this more demanding task, and indeed fared substantially worse than matched controls with moderate learning difficulties (MLD).

It seems to me that Russell's account of the contrast between a Sally-Anne task and the original false photo tasks (from Leekham and Perner (1991), Leslie and Thaiss (1992)) is incorrect. Firstly, in keeping track of the Sally-Anne story, no issue arises about the relative salience of my true belief and Sally's false one. What is salient to me is not my belief about the location of the chocolate. Rather it is the worldly situation of the chocolate's being in the cupboard. That is more salient than the past situation of the chocolate's being in the drawer. Secondly, it is misleading to talk of a comparison of salience between a three-dimensional representation (a doll in a yellow dress or a toy on a toy chair) and a two-dimensional one. The dimensionality is irrelevant. What is relevant is that the subject cannot see and never has seen the front of the photograph. What you can see is more salient than what you cannot see and have never seen. Seeing the back of the photograph is no more help than seeing Sally's head, when it comes to attending to the past situation or the content of a representation.

The executive demands are just the same in the Sally-Anne and the original photo tasks. In all cases, to succeed one has to retain in memory the original situation (Sally puts the chocolate in the drawer, the animal is on a chair), recall the relevant relation between the original situation and the representer (Sally, the camera), while the situation alters before your eyes and attend to the recollected situation when answering a question about the content of a representation. 
Russell et. al.'s results did show that autistic children tended to find the modified photo task harder than the original photo task, while children with MLD did not. This adds confirmation to the view that autism involves weak executive function as well as weak mindreading. But the results do not relate to the issue of what explains autistic subject's poor performance on Sally-Anne tasks. That would appear to be a specific problem in understanding the place of beliefs in the causal nexus.

At the end of the day, then, autism cannot be explained as a purely non-FP deficit.

\section{REFERENCES}

Ariew, A., (1996) "Innateness and Canalization" Proceedings Philosophy of Science (Proceedings), 1927, Cleveland, Ohio.

S. Baron-Cohen, (1994) "How to build a baby that can read minds: Cognitive mechanisms in mindreading" Cahiers de Psychologie Cognitive/ Current Psychology of Cognition 13:513-552

Baron-Cohen, S., and Swettenham, J., (1997) "Theory of mind in autism: its relationship to executive function and central coherence" in D. Cohen and F. Volkmar (eds) Handbook of Autism and Pervasive Developmental Disorders $2^{\text {nd }}$ edition Job Wiley and Sons.

Baron-Cohen, S., Wheelwright, S., Stone, V.E. \& Rutherford, M.D. (1999). "A mathematician, a physicist and a computer scientist with Asperger Syndrome: Performance on folk psychology and folk physics tests" Neurocase, 5, 475-483.

Bloom, P., (2004). Descartes' Baby: How the science of child development explains what makes us human. New York: Basic Books.

Boyer, P., (2003) "Religious thought and behaviour as by-products of brain function" Trends in Cognitive Science 73

Carruthers, P., (1992) Human Knowledge and Human Nature. Oxford: Oxford University Press.

Chomsky, N, (1988) Language and Problems of Knowledge Cambridge MA: MIT Press.

Chomsky N., (1986) Knowledge of Language New York: Praeger

Cowie, F., 1999 What's Within: Nativism Reconsidered Oxford University Press Oxford.

Crain, S., and Pietroski, P., (2002) 'Why Language Acquisition is a Snap' The Linguistic Review 19, 1-2. 
Csibra, G., (2003) "Teleological and Referential Understanding of Action in Infancy" Royal Society lecture, published online: http://www.itm.bme.hu/ktk/csaba/kszeminarium/csibra.2003.pdf.

Fodor. J., (1987) Psychosemantics Cambridge: The MIT Press.

Frith, U., and Happe, F., (1994) "Autism: Beyond Theory of Mind." Cognition, 50, pp.115-132

Gerrans, P., (2002) "The Theory of Mind Module in Evolutionary Psychology" Biology and Philosophy 17: $305-321$

Gopnik, A., and Wellman, H., (1992) "Why the Child's Theory of Mind Really is a Theory" Mind and Language 7 145-71

Gopnik, A., and Melktzoff, A., (1997) Words, Thoughts and Theories Cambridge MA: The MIT Press.

Griffiths, P. E. 1997. What Emotions Really Are: The Problem of Psychological Categories. Chicago: University of Chicago Press.

Griffiths, P. 2002. "What is Innateness?," The Monist, 70-85.

Hobson R.P. (2002) The Cradle of Thought Macmillan

Karmiloff-Smith, A., (1998) "Development itself is the key to understanding developmental disorders" Trends in Cognitive Science 2 389-398.

Kuhlmeier, V. A., Wynn, K. \& Bloom, P, (2004) Bloom, Wynn "Do 5-Month-Old Infants See Humans as Material Objects?" Cognition, 94, 95-103.

Laurence and Margolis 2001 "The Poverty of Stimulus Argument" The British Journal for the Philosophy of Science 52(2):217-276

Leekham, S. and Perner, J., (1991) "Does the autistic child have a 'metarepresentational' deficit?" Cognition 40 203-218

Leslie, A., (1994) "Pretending and Believing Issues in the Theory of TOM" Cognition 50 211-38.

Leslie, A., and Thaiss, L., (1992) "Domain specificity in Conceptual Development: Neuropsychological evidence from autism" Cognition 43 225-251

Meltzoff, A. \& Gopnik, A., (1993) "The Role of Imitation in Understanding Persons and Developing a 
Theory of Mind" in Baron-Cohen, S., Tager-Flusberg, H., and Cohen D. J., Understanding Other Minds: Perspectives from Autism New York: Oxford University Press 335-366

Nichols, S., and Stich, S., (2003) Mindreading: An Integrated Account of Pretence, Self-Awareness and Understanding Other Minds Oxford University Press: Oxford.

Pinker, S., (1994) The Language Instinct London: Penguin

Pullum, J., and Scholz, B., (2002) "Empirical Assessment of Stimulus Poverty Arguments" The Linguistic Review 19, 1-2

Russell, J., ed Autism as an Executive Function Disorder

Russell, J., Saltmarsh, R. \& Hill, E. (1999) 'What Do Executive Factors Contribute to the Failure on False Belief Tasks by Children with Autism?' Journal of Child Psychology and Psychiatry and Allied Disciplines, 40, 6, 859-868

Samuels R. (2002) "Nativism in Cognitive Science" Mind and Language, 17, 3, 233-265.

Schultz., L and Gopnik., (2004) A "Causal learning across domains" Developmental Psychology 402 167176

Sterelny, K., 2003 Thought in a Hostile World: The Evolution of Human Cognition Blackwell.

Varley, R. (1998). "Aphasic language, aphasic thought: propositional thought in an apropositional aphasic." In P. Carruthers \& J. Boucher (Eds.) Language and Thought: interdisciplinary themes. Cambridge: Cambridge University Press.

van der Lely, H. K. J., \& Stollwerck, L. (1996). A grammatical specific language impairment in children: An Autosomal Dominant Inheritance? Brain \& Language. 52, 484-504

van der Lely, H.K.J., Rosen, S., \& Adlard, A., (2004) "Grammatical language impairment and the specificity of cognitive domains: Relations between auditory and language abilities." Cognition 94, $167-183$ 
van der Lely, H.K.J., Rosen,S. and McClelland, A. (1998) Evidence for a grammar-specific deficit in children. Current Biology, 8:1253-1258

Waddington, C. H. (1940) Organizers and Genes. Cambridge: Cambridge U. Press. 